\title{
Automatic Target Recognition with Unknown Orientation and Adaptive Waveforms
}

\author{
Junhyeong Bae \\ Department of Electrical and Computer Engineering \\ University of Arizona \\ 1230 E. Speedway Blvd, Tucson, Arizona 85721 \\ dolbit@email.arizona.edu
}

\author{
Nathan A. Goodman \\ Department of Electrical and Computer Engineering \\ University of Arizona \\ 1230 E. Speedway Blvd, Tucson, Arizona 85721 \\ goodman@ece.arizona.edu
}

\begin{abstract}
In previous work, we have demonstrated the utility of a feedback loop for enabling optimized transmit pulse shaping in radar target recognition. This previous work was based on low-fidelity target models, but in this paper, we demonstrate the closed-loop, adaptive-waveform approach applied to highfidelity target model signatures generated by commercial electromagnetic FDTD software. We also incorporate the radar equation into our models for us in the waveform design procedure. Because SNR varies with range, so do our optimized waveforms for target recognition. Constant-modulus waveform constraints are enforced, and a template-based classification strategy is used.
\end{abstract}

\section{INTRODUCTION}

Cognitive radar [1] uses an adaptive transmitter to transmit customized waveforms. At each transmission, the adaptive transmitter updates its waveform based on the radar's objectives, a probability model for previous measurements, and other prior information. Waveform customization can refer to both the temporal structure of the waveform as well as the transmit beampattern. Because the adaptive transmitter exploits previous measurements, we can view cognitive radar as having a feedback loop between the radar receiver and transmitter. This feedback loop delivers analyzed information from previous radar measurements to the radar transmitter in the form of updated prior information. In this paper we focus on the customization of a waveforms temporal structure in order to perform automatic target recognition (ATR) with reduced transmit power and/or at longer range.

The goal of ATR is to identify an object that is observed by the radar system. In our earlier work [2], an adaptively shaped temporal waveform was applied to a target identification scenario. This work showed that a radar performing ATR according to cognitive radar principles uses fewer radar resources (i.e., reduced power or energy) and makes a fast decision with low error rate. Cognitive radar can also be applied to make better use of the radar timeline in detection and tracking scenarios. In [3], a cognitive-radarbased technique for adaptive beamsteering was implemented.
This closed-loop approach to beamsteering has been demonstrated to improve detection time. In [4], cognitive tracking radar was implemented. The transmit waveform was selected from a prescribed library according to information collected by the receiver, and the cognitive tracking radar was shown to outperform conventional radar. In [5], cognitive radar with knowledge-aided (KA) processing was proposed.

We extend our previous work in the area of ATR with two contributions in this paper. First, we use new high-fidelity target signatures. In previous work, our target signatures were generated from simple arbitrary target outlines and handplaced scatterers, which allowed the signatures to vary with rotation of the target. In this paper, we use commercial EM software (XFdtd, by Remcom) and publicly available target $\mathrm{CAD}$ models to calculate target signatures versus angle. Second, the radar equation is incorporated directly into the target signatures to model propagation loss. Therefore, the radar equation affects the target signature spectral strengths. Because optimized waveform design is SNR-dependent, using the radar equation in the target signature model affects our waveform design procedures. The performance of closed-loop radar can then be considered as a function of target range.

This paper is organized as follows. In Section II, we present the problem statement and signal model. In Section III, we describe the target signature model. In Section IV-A, we show the waveform design technique and incorporation of the radar equation to the design technique. In Section IV-B, we summarize constant-modulus waveform constraints. In Section $\mathrm{V}$, we describe the decision-making procedure and probability updates based on Bayes' Theorem. In Section VI, we show simulation results, and in Section VII, we make our conclusions.

\section{PRoblem STATEMENT AND SIGNAL MODEL}

A monostatic radar system with a matched illumination waveform is applied to the target recognition scenario in the presence of additive white Gaussian noise (AWGN). The basic problem formulation is similar to our previous work [6] 
and summarized below. It is assumed that a target has already been detected and is known to be one of $M$ possible target types. The target has a continuum of target signatures as a function of azimuth and elevation angles. For simplicity, here we hold the elevation angle fixed and consider only azimuth rotation. Consider a linear target model as follows. When the radar waveform $s(t)$ is transmitted, the radar received signal $y(t)$ is denoted as

$$
y(t)=g(t) * s(t)+n(t)
$$

where $g(t)$ is the azimuth-varying target signature of the unknown target, $*$ is the convolution operator, and $n(t)$ is AWGN with power $\sigma_{n}^{2}$.

A discrete-time version of the signal model is necessary to implement as a computer simulation. In discrete-time notation, the target signature is represented by a length- $L_{g}$ vector $\mathbf{g}$, the waveform is denoted as a length- $L_{s}$ vector $\mathbf{s}$, the noise is defined as a length- $L_{n}$ vector $\mathbf{n}$, and the received signal is represented by a length- $L_{y}$ vector $\mathbf{y}$. To implement the convolution between waveform and target impulse response in the discrete-time model, a signal matrix $\mathbf{S}$ is defined as [7]

$$
\mathbf{S}=\left[\begin{array}{ccccc}
s(1) & 0 & \cdots & \cdots & 0 \\
s(2) & s(1) & \ddots & \cdots & 0 \\
\vdots & \vdots & \ddots & \ddots & \vdots \\
s\left(L_{s}\right) & s\left(L_{s}-1\right) & \cdots & s(1) & 0 \\
0 & s\left(L_{s}\right) & s\left(L_{s}-1\right) & \cdots & s(1) \\
\vdots & 0 & s\left(L_{s}\right) & \cdots & s(2) \\
\vdots & \vdots & 0 & \ddots & \vdots \\
0 & 0 & \cdots & 0 & s\left(L_{s}\right)
\end{array}\right] .
$$

Using the signal matrix and above definitions, the discretetime signal model is

$$
\mathbf{y}=\mathbf{S g}+\mathbf{n} .
$$

To handle target signatures that vary with azimuth angle, the azimuth angle is divided into $N_{g}$ uniformly sized angular sectors. Multiple target signatures are generated for angles within each sector, and the signatures are averaged to acquire a mean template for that sector. The mean templates of all $M$ target types are defined as $\overline{\mathbf{g}}_{i}(t), i=1, \ldots, N_{g}, \ldots, M N_{g}$. When the number of sectors $N_{g}$ is large, the size of each sector is small, and the mean-template for each sector is a good representation of the target signatures across the sector. However, computational complexity increases when we must consider many sectors, especially in cognitive radar where probabilities associated with each sector will be updated after each transmission. Thus, the sector size should be based on both required accuracy and system complexity. To employ prior information about the target orientation (derived, for example, from target bearing information), the target orientation at the beginning of the recognition phase is assumed known to within a few angular sectors.

To set up the target recognition problem in terms of hypothesis testing, we define a single hypothesis as corresponding to a single angular section within a single target type. Thus, for the sake of feeding information back to the transmitter and optimizing the waveform design, each angular sector is treated as a different hypothesis. As measurements are received, the probabilities associated with each target/sector combination are updated to reflect what has been learned. But to do this, we need to define a probability density function (pdf) of the radar received signal for each target/sector hypothesis. One potential distribution is Gaussian, which might be able to capture both the mean template for a sector as well as the variation of the signatures around the mean for that sector. Unfortunately, a multivariate normality test applied to the XFdtd target signatures [8] over a sector showed that Gaussian was not representative, even as an approximate distribution. Thus, instead of making a Gaussian assumption for the target signatures, the signatures are treated as constant across a sector, resulting in a deterministic model with the mean-template $\overline{\mathbf{g}}_{i}$ for each sector. Since the waveform $\mathbf{s}$ and target template (given a particular target and sector) are deterministic, and the noise $\mathbf{n}$ is AWGN, the received signal $\mathbf{y}$ is Gaussian. The pdf of the complex received signal given the $i^{\text {th }}$ target/sector hypothesis is defined as

$$
p\left(\mathbf{y} \mid H_{i}\right)=\frac{1}{\left(\pi \sigma_{n}^{2}\right)^{N}} \exp \left(\frac{-1}{\sigma_{n}^{2}}\left(\mathbf{y}-\boldsymbol{\mu}_{y, i}\right)^{H}\left(\mathbf{y}-\boldsymbol{\mu}_{y, i}\right)\right)
$$

where $\boldsymbol{\mu}_{y, i}=\mathbf{S} \overline{\mathbf{g}}_{i}$ is the mean of the received signal under the $i^{\text {th }}$ target/sector hypothesis and $(\cdot)^{H}$ is the conjugate transpose operator. The mean signal $\boldsymbol{\mu}_{y, i}$ is waveformdependent and must be updated as the transmit waveform changes.

\section{TARGET MODEL}

In prior work [6], our target models consisted of arbitrary target outlines with scattering centers placed at various locations along the outlines. This model allowed the rotation of the target to affect the resulting target signatures. However, the models were admittedly low-fidelity. Here, we use a 3D commercial electromagnetic (EM) simulator, XFdtd, to calculate high-fidelity target signatures. The XFdtd software was provided by Remcom. The setup for generating the signatures is as follows. We used scaled 3D target CAD models in the XFdtd simulations. A monostatic radar was located in far-field. A broadband waveform was transmitted to the target and the reflected signal was stored. The procedure was repeated at many different aspect angles to create a received signal library. With each reflected signal, we calculated frequency-domain target transfer functions according to

$$
G(f)=Y(f) / S(f)
$$




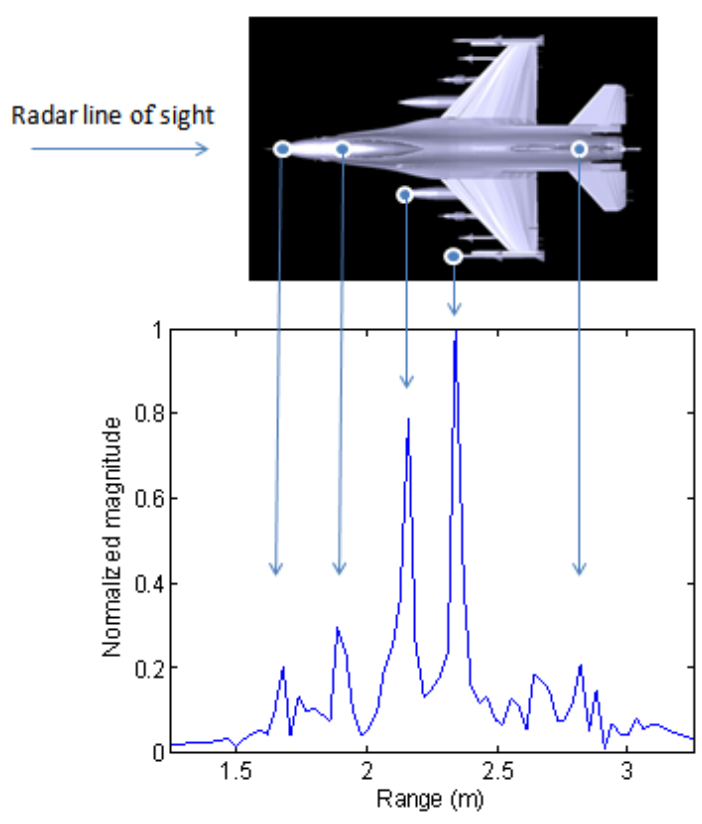

Figure 1. Head-on target signature of F-16 aircraft.

where $G(f), Y(f)$, and $S(f)$ are the Fourier transforms of target signature, the radar received signal, and the wideband transmitted waveform, respectively. The bandwidth of the transmitted pulse in this signature generation phase was significantly larger than the maximum waveform bandwidth for any of the ATR simulations that used the signatures. For the cognitive-radar ATR simulations, a section of the target transfer function corresponding to the radar transmission band was extracted from the wideband function in (5). The timedomain target signature was then generated from the inverse Fourier transform of the resulting bandlimited target transfer function.

Two examples of target signatures generated by XFdtd are shown in Figures 1 and 2. A monostatic radar is located slightly above the target's horizontal plane at $20^{\circ}$ elevation. A wideband pulse with $5 \mathrm{GHz}$ bandwidth was simulated via XFdtd. Figure 1 shows the head-on target signature for an F16. The length of F-16 CAD model is $1.445 \mathrm{~m}$ (approximately a 10:1 scale model). The peaks of the target signature correspond to the location of scatterers in the CAD model. The first peak corresponds to the tip of the nose and the second peak corresponds to the canopy of aircraft. The two largest peaks correspond to the largest under-wing missiles. The last peak is from the tail of the aircraft. The length of target signature between the first peak and the last peak is slightly smaller than the CAD length, because the radar is above the target and the reflection from the tail happens at the leading edge of the tail. Figure 2 shows the target signature of an A-10 CAD model at $30^{\circ}$ azimuth. The length of A-10 CAD model is $0.817 \mathrm{~m}$. The first and the last small peaks correspond to the nose and tail of the A-10, respectively. The four big peaks are generated by the two

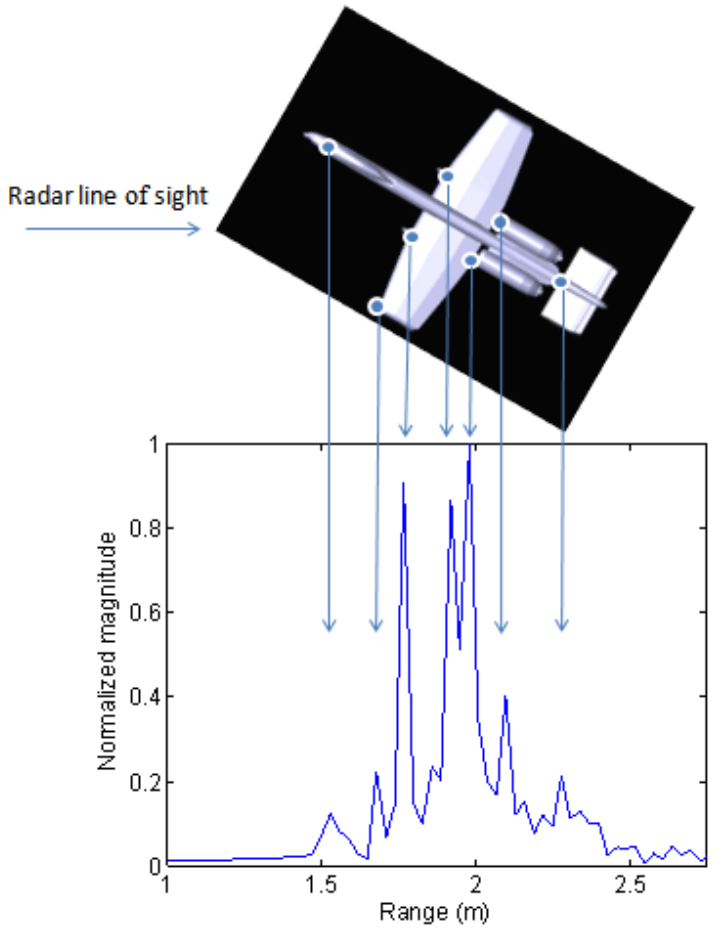

Figure 2. Azimuth angle $30^{\circ}$ target signature of A-10 aircraft.

under-wing landing-gear housings and two engines. In XFdtd, the propagation of the EM wave in space is calculated according to Maxwell's equations, which provides much more representative target signatures than we have used previously.

\section{RADAR WAVEFORM DESIGN}

\section{A. Waveform design and the radar equation}

The waveform design technique that we use here is based on maximization of mutual-information. The technique is adapted from the analysis in [9] and summarized below. We assume that an ensemble of target impulse responses exists. We also assume that the radar waveform has several constraints (energy, time, and frequency). For a Gaussian target ensemble, the waveform that maximizes the mutual information between the radar received signal and the (Gaussian) ensemble of target impulse responses can be found according to [9]

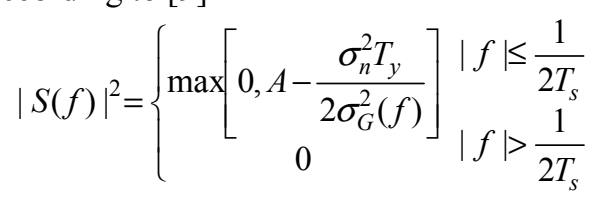

where the ensemble's spectral function is defined as $\sigma_{G}^{2}(f)=E\left\{|G(f)-E\{G(f)\}|^{2}\right\}, G(f)$ is the Gaussian target transfer function, and $T_{s}$ is the sampling interval of the signals. The total energy in the waveform is controlled by the scalar value $A$, such that 


$$
E=\int_{-1 / 2 T_{S}}^{1 / 2 T_{S}} \max \left[0, A-\frac{\sigma_{n}^{2} T_{y}}{2 \sigma_{G}^{2}(f)}\right] d f .
$$

As mentioned, the above design technique is based on a Gaussian ensemble, which we do not have. Fortunately, the spectral variance function can be extended for a finite number of discrete target hypotheses according to [2]

$$
\sigma_{G}^{2}(f)=\sum_{i=1}^{M N_{g}} P_{i}\left|\bar{G}_{i}(f)\right|^{2}-\left|\sum_{i=1}^{M N_{g}} P_{i} \bar{G}_{i}(f)\right|^{2}
$$

where $\bar{G}_{i}(f)$ is the Fourier transform of the $i^{\text {th }}$ mean target template. This spectral variance can be substituted in (6), and the waveform spectrum is found according to the waterfilling technique [9]. In this technique, the desired waveform energy spectrum is acquired by inverting the function $A-\sigma_{n}^{2} T_{y} / 2 \sigma_{G}^{2}(f)$ and pouring energy into the lowest parts of the inverted function until the allowable energy is gone. The amount of energy that is poured into each spectral component determines the waveform's magnitude spectrum. The phase of the waveform is an additional design variable that may be used to meet other design constraints, such as a constant modulus constraint.

As seen in (6), the optimum waveform depends on the noise power as well as the strength of the target ensemble (through the spectral variance). When SNR is low, the waveform defined by (6) will tend to have energy in only a couple narrow spectral bands. When SNR is high, the waveform becomes diversified and spreads its energy over the allowable band. Because the waveform design is SNRdependent, it is important to factor the radar equation into the signal model. The power, $P_{r}$, of the return signal from the target at the radar receiver can be calculated by the radar equation according to [10]

$$
P_{r}=\frac{P_{T} G_{a}^{2} \lambda^{2} \sigma}{(4 \pi)^{3} R^{4}}=P_{T} \sigma C=P_{T} \sigma_{e f f}
$$

where $P_{T}$ is the radar transmit power, $G_{a}$ is the antenna gain, $\lambda$ is the operating wavelength, $\sigma$ is radar cross section, and $R$ is the range between radar and target. The variable $C$ can be incorporated into $\sigma$ to represent an effective radar cross section that varies with range, and this effective radar cross section can be used to properly scale the target signature library. Therefore, propagation loss is factored into the spectral variance function above, which then affects the waveform design.

In this work, we scale the target template libraries such that the average level of the magnitude of all target transfer functions for a particular target is equal to the average square root of RCS for that target. In other words, suppose that a target has an RCS of $\sigma$, then we set the target transfer function scaling such that

$$
\overline{|G(f)|}=\sqrt{C} \mathrm{E}[\sqrt{\sigma}]
$$

This normalization ensures that for a narrowband waveform centered around frequency $f_{0}$, we have a constant-valued transfer function over the waveform bandwidth with average magnitude equal to

$$
\mathrm{E}\left[\left|G\left(f_{0}\right)\right|\right]=\sqrt{C} \mathrm{E}[\sqrt{\sigma}] .
$$

The received waveform will then be

$$
y(t)=\sqrt{\frac{G_{a}^{2} \lambda^{2}}{(4 \pi)^{3} R^{4}}} \mathrm{E}[\sqrt{\sigma}] s(t-2 R / c)+n(t) .
$$

With the scaling of the radar range equation incorporated into the target signature, the signal model now fits the form of (1) as required by the mutual information waveform design method.

\section{B. Waveform constraint}

Any practical radar system has a peak power limitation, so the temporal radar waveform should be designed and operated under this limitation. Thus, a constant modulus [11] constraint on the radar waveform is necessary to operate the radar system efficiently.

The technique used here to construct a constant-modulus signal with a prescribed Fourier transform magnitude is based on iterative magnitude and amplitude projections. The technique is presented in [11] and summarized below. The set of functions $\{v(t)\}$ with equal Fourier transform magnitude $F(w)$ over the frequency set $\Psi$ is denoted as $D_{M}$. Then, we can define a magnitude projection operator $P_{M}$ that projects an arbitrary function $x(t)$ to nearest point on $D_{M}$. Assuming the Fourier transform of $x(t)$ is $X(w)=|X(w)| e^{j \Omega(w)}$, the magnitude projection procedure is represented by

$$
P_{M} x(t)=\left\{\begin{array}{lc}
F(w) e^{j \Omega(w)}, & w \in \Psi \\
X(w), & w \in \Psi^{\prime}
\end{array} .\right.
$$

The set of functions $\{v(t)\}$ with positive constant value $B$ over the temporal duration $T$ is represented by $D_{A}$. Then, we can also define an amplitude projection operator $P_{A}$ that projects an arbitrary function $x(t)$ to closest point on $D_{A}$. Assuming $x(t)$ is equal to $a(t) e^{j \phi(t)}$, the amplitude projection is defined as

$$
P_{A} x(t)=\left\{\begin{array}{c}
B e^{j \phi(t)}, \quad t \in T \\
x(t), \text { otherwise }
\end{array} .\right.
$$

The above magnitude and amplitude projection is performed iteratively according to

$$
x_{k+1}(t)=P_{A} P_{M} x_{k}(t)
$$

where $x_{k}(t)$ is the arbitrary function after $k^{\text {th }}$ projection. After many iterations, the function $x(t)$ maintains exact constant modulus amplitude, but has a Fourier transform magnitude that approximates the desired Fourier magnitude. 


\section{FIXED NUMBER OF ITERATIONS AND BAYES’ THEOREM}

We adopt a procedure whereby a classification decision is made after transmitting a fixed number of optimized waveforms. Therefore, the number of transmissions is fixed in advance. At each transmission, the likelihoods are formed and the probabilities associated with each target/sector combination are updated. The expression of the $i^{\text {th }}$ hypothesis likelihood after the $k^{\text {th }}$ transmission depends on joint pdf of the received signals on all transmissions. However, since the radar waveform and target signature are modeled as deterministic, and the white Gaussian noise samples are uncorrelated and independent, the measurement data are statistically independent. Then, the joint pdf of the data on all transmissions can be accumulated to update the likelihood of the $i^{\text {th }}$ hypothesis according to

$$
p\left(H_{i} \mid \mathbf{y}_{k}\right) \propto p_{i 1}\left(\mathbf{y}_{1}\right) p_{i 2}\left(\mathbf{y}_{2}\right) \cdots p_{i k}\left(\mathbf{y}_{k}\right) P_{i}
$$

where $p_{i k}\left(\mathbf{y}_{k}\right)$ is the pdf of the $k^{t h}$ received signal for the $i^{\text {th }}$ hypothesis, $\mathbf{y}_{k}$ is the received signal due to $k^{\text {th }}$ transmission, and $P_{i}$ is the probability of the $i^{t h}$ hypothesis prior to any transmissions. The final decision is made after the number of transmission reaches the pre-defined iteration limit. The hypothesis $H_{i}$ corresponding to the highest likelihood is the final decision.

In the closed-loop radar system, the waveforms are updated at each transmission. To update the waveform, the hypothesis probabilities are update by Bayes' theorem according to (16) (except for a scaling factor that ensures the probabilities sum to unity). The updated probabilities are substituted into (8) to update the radar waveform.

\section{RESULTS}

We performed a computer simulation of a radar target recognition scenario based on the techniques above. We have two plane targets (F-16 and A-10). We assume that the elevation angle between the horizontal plane of the target and the radar line of sight is $20^{\circ}$, such that the monostatic radar is a little above the target. We consider azimuth angles over a $90^{\circ}$ range from head-on to broadside of each target. We generated target signatures at every $0.1^{\circ}$ interval. Since we know the actual size of these targets from the literature, we use a scaling factor to adjust the sampling interval and bandwidth of the original CAD-based XFdtd target signatures to the actual target size, approximately. Table I shows the lengths and scaling factors. The target signatures are grouped into $1^{\circ}$ sectors. The target signatures within each uniform angular sector are averaged to generate a mean-template. We assume that we have prior knowledge about the velocity and bearing information of a target. Thus, for a given trial of the ATR simulation, we can narrow down the possible target angular sectors to two adjacent mean-templates for each target. We treat each sector as a hypothesis, so we have four hypotheses in total for a given trail. For randomly selected
TABLE I. TARGET LENGTHS AND SCALING FACTORS

\begin{tabular}{|c|c|c|c|}
\hline Targets & Actual length(m) & CAD length(m) & Scaling factor \\
\hline F-16 & 15.06 & 1.445 & 10 \\
\hline A-10 & 16.26 & 0.817 & 20 \\
\hline
\end{tabular}

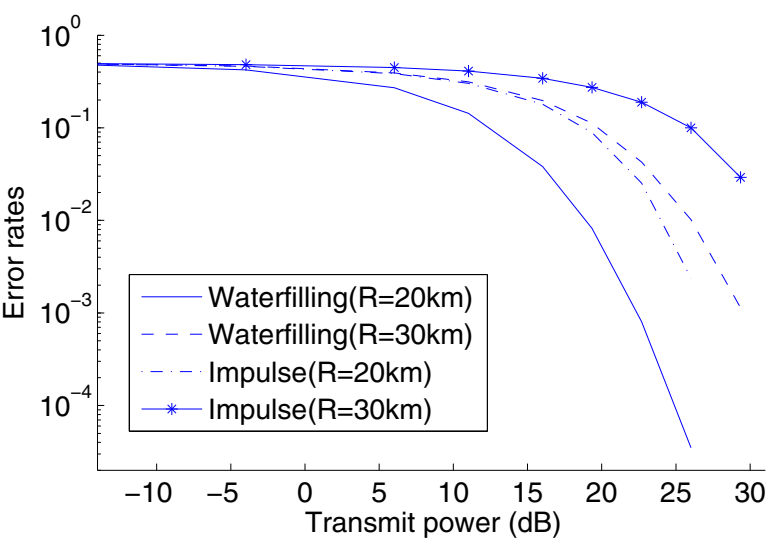

Figure 3. Error rates versus transmit power for fixed number of transmissions.

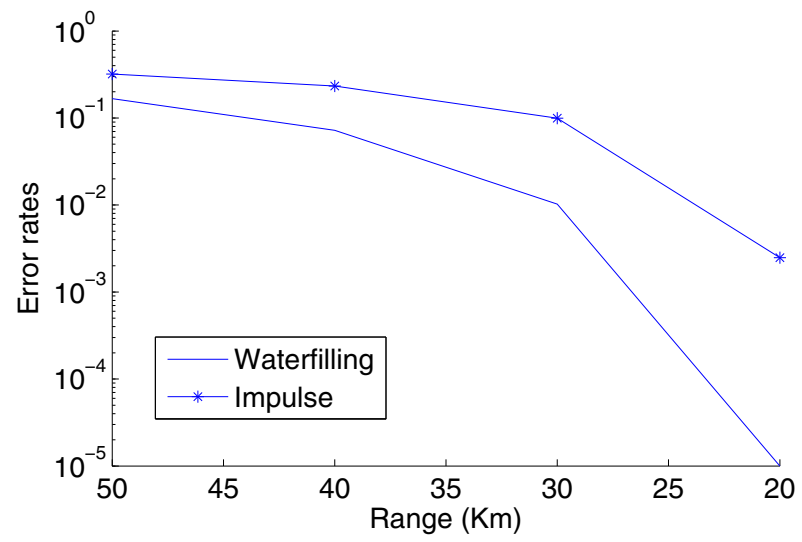

Figure 4. Error rates versus range for fixed number of transmissions.

target angles that do not fall on the $0.1^{\circ}$ increments, we generate the true target signature by a weighted average of the two adjacent target signatures. We compare the performance of two waveforms: the information-based waveform realized via the spectral variance strategy over a $250-\mathrm{MHz}$ bandwidth, and a $250-\mathrm{MHz}$ wideband impulse. We use the radar equation to compare the performance of the waveforms based on the range between radar and target. The radar parameters used were an antenna gain of $30 \mathrm{~dB}$ at $\mathrm{S}$ band, a noise temperature of $290 \mathrm{~K}$, and an average total RCS for each target of $1 \mathrm{~m}^{2}$. Five waveforms were transmitted before making a decision. We ran 200,000 Monte Carlo trials (over noise realizations and orientation angle), and counted the number of incorrect decisions to compute error rates. 
Figure 3 shows the error rates versus transmit power. The information-based waveform performs better than the wideband waveform for the same range because the information-based waveform puts energy into the frequency bands where spectral discrimination is strong. The radar return signal from farther range has lower signal-to-noise (SNR), so the error rates are higher. The two waterfilling waveforms (for $R=20 \mathrm{~km}$ and $30 \mathrm{~km}$ ) at error rate 0.01 are shift by approximately $7 \mathrm{~dB}$. Because the ratio between these distances is $30 / 20=1.5$, and the received power has a $1 / R^{4}$ relationship to target range, $10 * \log _{10}\left(1 /(1.5)^{4}\right)=-7.0437 \mathrm{~dB}$, and the $7 \mathrm{~dB}$ shift is expected. The information-based waveforms require about $5 \mathrm{~dB}$ less power than the wideband waveforms at the same range. Figure 4 shows the error rates versus range when the transmit power is $26 \mathrm{~dB}$. In this case, the information-based waveform can achieve the same error rate as the wideband waveform, but at an approximately $30 \%$ increase in range.

\section{CONCLUSIONS}

We have simulated a closed-loop radar system for target recognition using high-fidelity target models calculated via commercial EM software and publicly available target CAD models. We also incorporated the radar equation into the target signatures as part of the waveform design process. The information-based waveform with a constant modulus constraint was compared to a flat-spectrum wideband waveform in a target recognition scenario. The two targets being classified were an F-16 and an A-10. The results show that the information-based waveform provides approximately $5 \mathrm{~dB}$ improvement in transmitted power for the same error rate, which translated to a more than $30 \%$ increase in recognition range.

\section{ACKNOWLEDGMENT}

The authors acknowledge support from the ONR via grant \#N000140910338. We are also very grateful to Remcom for providing their XFdtd software.

\section{REFERENCES}

[1] S. Haykin, "Cognitive radar: a way of the future," IEEE Sig. Proc. Mag., vol. 23, no. 1, pp. 30-40, Jan. 2006.

[2] N.A. Goodman, P.R. Venkata, and M.A. Neifeld, "Adaptive waveform design and sequential hypothesis testing for target recognition with active sensors," IEEE J. Selected Topics in Signal Processing, vol. 1, no. 1, pp. 105-113, June, 2007.

[3] P. Nielsen and N.A. Goodman, "Integrated detection and tracking via closed-loop radar with spatial-domain matched illumination," in Proc. 2008 International Conference on Radar, Adelaide, Australia, pp. 546551, Sept. 2008.

[4] S. Haykin, A. Zia, I. Arasaratnam, and Y. Xue, "Cognitive tracking radar," 2010 IEEE Radar Conference, pp 1467-1470, Washington DC., May 2010.

[5] J.R. Guerci, "Cognitive radar: a knowledge-aided fully adaptive approach," 2010 IEEE Radar Conference, pp 1365-1370, Wasington DC., May 2010.

[6] J. Bae, N. A. Goodman, "Evaluation of modulus-constrained matched illumination waveform for target identification," 2010 IEEE Radar Conference, pp 871-876, Washington DC., May 2010.

[7] D.A. Garren, M. K. Osborn, A. C. Odom, J. S. Goldstein, S. U. Pillai, and J. R. Guerci, "Enhanced target detection and identification via optimized radar transmission pulse shape," Proc. IEEE, vol. 148, no. 3, pp. 130-138, Jun. 2001.

[8] S.P. Smith and A. K. Jain, "A test to determine the multivariate normality of a dataset,". IEEE Transactions on Pattern Analysis and Machine Intelligence, vol. 10, no. 5, pp 757-761, Sep. 1988.

[9] M.R. Bell, "Information theory and radar waveform design," IEEE Trans. Info. Theory, vol. 39, no. 5, pp. 1578-1597, Sept. 1993.

[10] D.R. Wehner, "High-resolution radar," Artech House Publishers, Boston, 1995.

[11] S.U. Pillai, K.Y. Li, and H. Beyer, "Construction of constant envelope signals with given Fourier Transform magnitude," 2009 IEEE Radar Conference, Pasadena, California, USA, May 4-8, 2009. 